\title{
Food literacy predictors and associations with physical and emergent literacy in pre-schoolers: results from the Training-to-Health Project
}

\author{
Garden Tabacchi ${ }^{1, *}$ (1), Giuseppe Battaglia ${ }^{1}$, Marianna Alesi ${ }^{1}$, Antonio Paoli ${ }^{2}$, \\ Antonio Palma ${ }^{1}$ and Marianna Bellafiore ${ }^{1}$ \\ 'Department of Psychological, Pedagogical, Educational Science and Human Movement, University of Palermo, Via \\ Giovanni Pascoli 6, 90144 Palermo, Italy: ${ }^{2}$ Department of Biomedical Sciences, University of Padova, Padova, Italy
}

Submitted 22 January 2019: Final revision received 8 May 2019: Accepted 23 May 2019

\begin{abstract}
Objective: Food literacy (FL) is a crucial need encompassing basic literacy and fundamental movement skills. The present study aimed to investigate the FL level in pre-schoolers and to evaluate the effect of potential predictors and the associations with gross motor and emergent literacy skills.

Design: Cross-sectional study conducted within the Training-to-Health Project. Setting: Kindergartens ( $n$ 21) in the Palermo City Council, Italy.

Participants: Pre-school children aged 3-6 years $(n$ 921) followed education sessions on nutritional topics, practical activities and compiled prearranged sheets. FL was assessed by the five-domain toolkit 'preschool-FLAT'; gross motor and emergent literacy skills were assessed by the Italian version of the gross motor development test and the PRCR-2/2009, respectively. Correlation and regression analyses were performed to assess relationships between FL scores and gender, age, weight, height, BMI, gross motor and emergent literacy skills.

Results: Independent predictors $(\beta, \mathrm{SE})$ of higher FL were female gender (1.06, $0.315, P<0.01)$, older age $(0.08,0.019, P<0.001)$ and greater height $(0.13$, $0.03, P<0 \cdot 001)$. The adjusted coefficients were significant for quotient of gross motor development and in particular for the locomotor component $(0.03,0.01$, $P<0.01$ and $0.16,0.046, P<0.001$, respectively). Almost all associations with the emergent literacy skills were significant $(\beta=-0.02$ to $0 \cdot 47)$.

Conclusions: The study suggests that children raised in an environment where both cognitive and motor skills are enhanced can have better chances of increasing FL and success at school. Thus, the need for monitoring FL and its predictors since early age is highlighted.
\end{abstract}

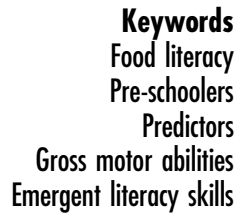

Food literacy

Pre-schoolers

Predictors

Emergent literacy skills
In the context of food and nutrition, 'knowledge' is commonly defined as the understanding of the health benefits of foods and nutrients ${ }^{(1)}$. Improving children's food knowledge and awareness is a major focus of community and school-wide intervention programmes that promote healthier lifestyles to prevent obesity ${ }^{(2-5)}$. Young children's knowledge about healthy foods may influence the formation of their eating behaviours ${ }^{(6)}$. The recent concept of food literacy (FL) has highlighted that not only nutrition knowledge but also skills and behaviours related to food and nutrition are important in defining and maintaining a healthy lifelong relationship with food ${ }^{(7)}$. FL is characterized as an individual's knowledge, skills and attitudes towards foods and food products; FL enables healthy food choice among children and can enable them to make their own choice of foods and rational decisions that are environmentally, economically and socially sustainable ${ }^{(8,9)}$. Moreover, different components of FL have been recognized, such as those according to Vidgen and Gallegos ${ }^{(8)}$ : planning and management skills (e.g. make rational choice of foods); selection; preparation (e.g. compose a meal with good taste based on availability); knowledge of cooking procedures, nature of foods, food hygiene and storage; eating (e.g. act according to needs and quantity); and understanding the social importance of food. 
FL encompasses abilities requiring basic literacy skills and motor abilities. For example, discriminating between healthy and non-healthy foods requires knowledge of what a food is, of what health is, and of the meaning of 'healthy' and 'non-healthy'; while being able to prepare meals, from kneading to peeling fruit, etc., requires motor abilities. In fact, emergent literacy skills include key components such as oral language development, which comprises vocabulary and listening; understanding the alphabetic code, which includes phonological/phonemic awareness and knowledge of the alphabet; and knowledge and understanding of print and its use ${ }^{(10)}$. Gross motor skills are those that require whole body movement and involve the large muscles of the body to perform everyday functions (e.g. walking, running, doing simple self-care skills, etc.) and also include eye-hand coordination skills such as throwing, catching, kicking, etc. In turn, they can affect the ability to participate in fine motor skills (e.g. writing, drawing, cutting) and sitting upright to attend to class instruction, which then impacts on academic learning ${ }^{(11)}$. Moreover, emergent literacy skills and gross motor development are strong predictors of later literacy and motor achievement $^{(12-14)}$.

Although there is no existing literature specifically linking FL and emergent literacy/motor skills, the authors found it interesting to assess the interrelationships between all these aspects. The underlying concept of this intent draws on different anatomical and physiological studies indicating the existence of common areas of the central nervous system, and common underlying processes such as sequencing, monitoring and planning ${ }^{(15,16)}$. During several motor and cognitive tasks, co-activations between the prefrontal cortex, cerebellum and basal ganglia have been found, especially when a task is difficult or new, since a quick response is required and concentration is needed to perform the task ${ }^{(17)}$. This means that the prefrontal cortex, which is well known to be linked to cognition, is also important for motor activity through its interconnections with cortical and sub-cortical centres; and that the cerebellum, which plays a well-known role in motor movements, has an important function also in cognitive skills ${ }^{(17)}$. These concepts suggest that both abilities are to be assessed when monitoring pre-school children, or that both have to be taken into account when intervention programmes are envisaged within this population group.

Various tools for the assessment of food knowledge have been used in schoolchildren from elementary to high schools $^{(18-20)}$; however, few studies have measured food knowledge in pre-school children or have investigated factors (such as gender, age, socio-economic/cultural level, weight status, etc.) that can shape young children's food knowledge or that can influence food behaviours ${ }^{(6,21-26)}$. A recent review demonstrated that different interactive and task-centred techniques have been used to assess components of food and nutrition knowledge in pre-school children; they included mainly practical activities as they were mostly developed to evaluate interventions, but the need for additional research to develop refined, valid and reliable assessment tools was outlined ${ }^{(27)}$.

Also, the assessment of FL being a quite recent concept, little information about instruments and determinants can be found in the literature ${ }^{(28-31)}$. The lack of validated tools inhibits the ability to assess and monitor FL, to tailor and target programmes, and to allocate resources. For this reason, a new tool called 'preschool-FLAT' (Food Literacy Assessment Tool) was recently designed and validated for the assessment of FL in pre-schoolers within the Training-to-Health Project. The aim of the present study was to describe FL in pre-schoolers, including knowledge and skills related to food and nutrition, and to evaluate the effect of potential predictors and the associations with gross motor and emergent literacy skills.

\section{Methods}

\section{Study design and participants}

The current cross-sectional study was carried out within the Training-to-Health Project, financed by the Municipality of Palermo and conducted by the University of Palermo in $2016^{(32)}$. In this project, twenty-one kindergartens under the Palermo City Council administrative boundaries were recruited. The sampling design included all schools under the City Council in order to cover both central and peripheral areas of the city. An initial number of 1054 children's parents were invited to join the project and were provided with informed sheets; since 126 refused, a total of 928 signed informed consents (response rate 88.1\%) were obtained from the parents. Participating children were 3-6 years old and attended three classes (one class per age range: 3-4, 4-5 and 5-6 years). A team composed by professionals in the field of physical exercise science, nutrition, psychology, medicine, agronomy and biology carried out the project activities and the training sessions. The study was approved by the Ethical Board of the Azienda Ospedaliera Universitaria Policlinico Paolo Giaccone Palermo (Palermo 1, No. 02/2018) and followed the criteria stated in the Declaration of Helsinki.

\section{Anthropometric measurements}

Body weight was assessed using a Seca electronic scale (maximum weight recordable, $300 \mathrm{~kg}$; resolution, $100 \mathrm{~g}$; Seca Deutschland, Hamburg, Germany). Height was measured through the use of a stadiometer (maximum height recordable, $220 \mathrm{~cm}$; resolution, $1 \mathrm{~mm}$ ) with the child barefoot and standing upright. BMI was calculated as weight (in kilograms) divided by the square of height (in metres); the international cut-off points for BMI based on gender and age from Cole et al. ${ }^{(33)}$ were used to categorize classes of weight status into underweight, normal weight, overweight and obese. 


\section{Assessment of food literacy}

FL was quantified with the preschool-FLAT, a tool created by the Training-to-Health team and composed by five modules encompassing a total of sixteen items. These items were accurately selected by experts in different fields. The content validity of the construct was demonstrated to be good, with a content validity index for each item of 0.94 , and a content validity ratio for the total scale, according to Lawshe scores, of 0.88. The internal consistency of the sixteen sub-tests obtained by the 505 participating children was adequate, since Cronbach's $\alpha$ coefficient ranged from 0.7338 to 0.7637 , with an overall $\alpha$ equal to 0.7649 and the average inter-item covariance equal to $0 \cdot 1712$. Finally, the analysis of construct validity through structural equation modelling revealed a four-factor model fitting the data well (comparative fit index $=0.939$, rootmean-square error of approximation $=0.033$, incremental and parsimony fit indices). These four factors represent four domains encompassing weight/food/health, food/ environment, traditional foods and food groups/ meals; and according to the analysis they are well explained by the sixteen selected items ( $G$ Tabacchi, G Battaglia, A Paoli, A Palma and M Bellafiore, unpublished results).

These modules are aimed at assessing children's knowledge, attitudes and skills on different aspects of food, diet and nutrition: weight status/food, weight status/health (Module 1); food quality or quantity/health (Module 2); food/environment (organic foods/meals, fresh foods, seasonality of foods) (Module 3); traditional foods/ meals (Module 4); and food groups/meals and food pyramid (Module 5). The effectiveness of the tool in the improvement of nutrition knowledge and skills of preschoolers was previously evaluated in comparison with a control group that did not perform the five modules; the FL score of the intervention group was significantly higher than that of the control (mean score: $15 \cdot 1 v \cdot 7 \cdot 1$, $P<0.001$, unpaired Student's $t$ test), this demonstrating good discriminant validity of the tool ( $G$ Tabacchi, G Battaglia, A Paoli, A Palma and M Bellafiore, unpublished results).

The food activities were structured in one brief oral session, where the operators provided information to the class on the topic of the module, and a subsequent practical session, where students were invited to perform activities related to the previously explained arguments and compile the prearranged assessment sheets.

For each item, a 5-point scale (from 0 to 4) according to Likert was possible. A total score ranging from 0 to 20 , obtained by the sum of the scores of the single items, was generated to rank children in classes of food knowledge and skills. Crude scores were standardized into $Z$-scores to identify children ranking below and above 1 SD and classify them into low FL level and high FL level, respectively.

\section{Measurement of gross motor and emergent literacy skills}

The Italian version of the gross motor development test was used to assess locomotor ability and object control skills ${ }^{(34)}$. This test requires that children run $15 \mathrm{~m}$ as fast as possible, gallop for $10 \mathrm{~m}$, hop on one leg for $5 \mathrm{~m}$, jump forward, do a long jump, and take little jumps forward and laterally, in order to assess the locomotor abilities. To assess the object control skills, children are required to catch a ball with a tennis racket, bounce off the ball, catch a ball, kick the ball running, and throw a ball with the hand. The combination of these two skills is named quotient of gross motor development (QGMD). Children's performances were videotaped with a digital video camera and analysed by two previously trained observers who assigned scores. Each child performed three trials of each skill and acquired 1 point when a criterion performance was correct two out of three times or 0 points when a criterion was not observed or was used inappropriately two out of three times. The sum of scores obtained for each item, with a maximum total score of 48 , was standardized according to age. The sum of scores obtained for each criterion (maximum total score 48) was transformed into standard scores according to the age level of the child. We evaluated the gross motor development level based on QGMD scores suggested by the manual's instructions: 35-69 (very low motor ability, VL-MA); 70-79 (low motor ability, L-MA); 80-89 (under average motor ability, UA-MA); 90-110 (average motor ability, A-MA); 111-120 (over average motor ability, OA-MA); 121-130 (high motor ability, H-MA); and 131-165 (very high motor ability, VH-MA) ${ }^{(34)}$.

The PRCR-2/2009 is a battery of standardized tasks aimed at measuring general and specific prerequisites to later reading and writing abilities in kindergarten children $^{(35)}$. Four tasks were derived by the PRCR-2/2009 battery and used in the present study: (i) printed letters identification; (ii) objects naming; (iii) partially hidden objects naming; and (iv) pointed objects naming.

The printed letters identification task measured the visual analysis ability and the spatial orientation. It was composed of a sheet with twelve target letters printed on the left and four letters for each target (the target and three distractor letters) printed on the right. A child was required to recognize and cross the target letter. The number of errors for the task was recorded. The final score was obtained by adding together the number of errors.

The objects naming task measured the linguistic proficiency, the visual attention and the sequentiality of eye movements. It was composed of thirty objects in five sequences of six objects for each. The objects were for example animals (mouse, cat, chick), flowers, ice cream, sun, star, etc. A child was required to rapidly name the sequence of the objects from left to right and from the top to bottom. The number of errors and the execution time for the task were recorded. The final scores were the sum of errors and the time. 
The partially hidden objects naming task measured the linguistic proficiency, the visual attention and discrimination, and the sequentiality of eye movements. It was composed of three sequences of objects that appeared in the objects naming task, but the objects were overlapping and smaller. A child was required to rapidly name the sequence of the objects from left to right and from the top to bottom. The number of errors and the execution time for the task were recorded. The final scores were the sum of errors and the time.

The pointed objects naming task measured the visuo-perceptual ability to identify a figure from the background, the linguistic proficiency, the visual attention and discrimination, and the sequentiality of eye movements. It was composed of two sequences of overlapping objects that appeared in the partially hidden objects naming task, with four objects for each sequence marked by a dot at $15 \mathrm{~mm}$. A child was required to rapidly name the marked objects from left to right and from the top to bottom. The number of errors for the task was recorded. The final score was obtained by adding together the number of errors.

Both evaluations were carried out at the end of the project activities.

\section{Data analysis}

Distributions of variables were checked for normality before statistical analysis through the Shapiro-Wilk test. Sample characteristics were then described using mean and SD for continuous variables and number and percentage for categorical ones.

A non-respondent analysis was performed to compare the characteristics of children not completing all modules with those of respondents, through the unpaired Student's $t$ test for quantitative variables and the $\chi^{2}$ test for categorical outcomes.

A one-sample multivariate test of means was performed to assess the variance of the means of the single modules. The unpaired Student's $t$ test was used to estimate score differences by gender and school socio-economic environment. Bivariate correlation analyses corrected with Bonferroni and generating Pearson correlation coefficients ( $r$ ) were initially performed to assess associations of both overall and single module scores of FL with age, weight, height, BMI, gross motor and emergent literacy skills. Multiple linear regressions were subsequently performed to identify gender, age, weight, height, BMI, school socio-economic environment, gross motor and emergent literacy skills as possible independent predictors.

\section{Results}

A total of 921 pre-schoolers participated in the present study. Data on weight, height, FL scores, gross motor skills and emergent literacy skills were not complete for all 921
Table 1 Characteristics of the sample of pre-schoolers $(n$ 921) involved in the Training-to-Health Project, Palermo, Italy, 2016

\begin{tabular}{|c|c|c|c|}
\hline & $n$ & $\%$ & \\
\hline Gender & 921 & & \\
\hline Male & 501 & 54.4 & \\
\hline Female & 420 & $45 \cdot 6$ & \\
\hline School SEE & 921 & & \\
\hline Medium/low & 515 & 55.9 & \\
\hline Medium/high & 406 & $44 \cdot 1$ & \\
\hline Weight status & 758 & & \\
\hline Underweight & 77 & $10 \cdot 2$ & \\
\hline Normal weight & 504 & $66 \cdot 5$ & \\
\hline Overweight & 119 & $15 \cdot 7$ & \\
\hline \multirow[t]{2}{*}{ Obese } & 58 & $7 \cdot 7$ & \\
\hline & $n$ & Mean & SD \\
\hline Age (months) & 852 & $57 \cdot 0$ & $10 \cdot 39$ \\
\hline Weight $(\mathrm{kg})$ & 759 & 19.5 & 3.99 \\
\hline Height (cm) & 783 & $109 \cdot 4$ & 0.07 \\
\hline BMI $\left(\mathrm{kg} / \mathrm{m}^{2}\right)$ & 758 & $16 \cdot 1$ & $2 \cdot 27$ \\
\hline \multicolumn{4}{|l|}{ Food literacy scores } \\
\hline Overall food literacy & 505 & $15 \cdot 0$ & 3.80 \\
\hline Correct weight status & 830 & 2.9 & 1.25 \\
\hline Healthy foods/correct portions & 802 & $3 \cdot 2$ & $1 \cdot 11$ \\
\hline Organic/fresh foods seasonality & 766 & 3.0 & $1 \cdot 18$ \\
\hline Traditional foods/meals & 686 & 2.9 & 1.15 \\
\hline Meal composition/food pyramid & 793 & $2 \cdot 9$ & $1 \cdot 27$ \\
\hline \multicolumn{4}{|l|}{ Gross motor skills scores } \\
\hline QGMD & 837 & $123 \cdot 1$ & $19 \cdot 12$ \\
\hline Locomotor skills & 838 & $13 \cdot 8$ & 4.29 \\
\hline Control object skills & 839 & 14.0 & 3.39 \\
\hline \multicolumn{4}{|l|}{ Emergent literacy skills errors and time } \\
\hline Printed letters identification (errors) & 817 & $2 \cdot 7$ & $2 \cdot 60$ \\
\hline Objects naming (time, $s$ ) & 776 & $62 \cdot 9$ & $24 \cdot 36$ \\
\hline Objects naming (errors) & 775 & $1 \cdot 2$ & 1.88 \\
\hline Partially hidden objects naming (time, $s$ ) & 768 & 98.7 & 37.52 \\
\hline Partially hidden objects naming (errors) & 807 & 3.9 & 4.78 \\
\hline Pointed objects naming (errors) & 775 & 1.6 & 1.48 \\
\hline
\end{tabular}

SEE, socio-economic environment; QGMD, quotient of gross motor development.

owing to the fact that children were not present at school on the day of measurements or tests. Since not all children performed the activities and filled in the modules, data from the sum of all modules were obtained for a total of 505 pre-schoolers. The 416 children who did not complete all the five modules were here considered as non-respondents, and a supplementary analysis was conducted to compare their characteristics with those of respondents in order to detect any eventual dissimilarity that could influence FL results. Such analysis showed that there was no difference between the two groups in gender, age, school socio-economic environment, weight status, height, weight or BMI (see online supplementary material, Supplemental Table S1).

Table 1 shows the characteristics of the sample. Children had an overall FL mean score of $15 \cdot 0$ (SD 3.80). A onesample multivariate test of means demonstrated that the mean scores of the single modules were statistically different (Hotelling $F_{(5,473)}=728.43, P<0.001$ ); the best mean score was shown in the Module 2; mean scores of the other modules were about 3 (Table 1 ). The $Z$-score standardization showed that $20.0 \%$ of participants were at a low 
level of FL, $59.3 \%$ at medium level and $20.7 \%$ at high level. With regard to gross motor abilities, we found that children had mean locomotor and control object skills of 13.8 (SD 4.29) and 14.0 (SD 3.39), respectively, and a mean QGMD score of 123.1 (SD 19.12) showing high motor ability (Table 1).

Concerning the emergent literacy skills, children had a mean score (errors) of 2.7 (SD 2.60) on the task of printed letters identification, of 1.2 (SD 1.88) on the task of objects naming, of 3.9 (SD 4.78) on the task of partially hidden objects naming and of 1.6 (SD 1.48) on the task of pointed objects naming (Table 1 ). Moreover, they executed the task of objects naming in a mean time of 62.9 (SD 24.36) s and the task of partially hidden objects naming in a mean time of 98.7 (sD 37.52) s (Table 1).

The means of errors on the printed letters identification and partially hidden objects naming tasks are quite similar to those reported in the handbook, of 2.14 (SD 2.22) and 3.03 (SD 3.93), respectively. Means of time on the objects naming and partially hidden objects naming tasks were similar to those reported in the handbook, of 64.89 (SD 58.06) s and 91.16 (SD 23.62) s, respectively.

The mean comparison test showed that gender and FL overall score were not correlated, while in Modules 3 and 4 females had better performances than males (Table 2). The school socio-economic environment was correlated to FL overall score and the score of Modules 2 and 4 in the mean comparison test (Table 2). Weight status was shown not to influence both the overall and the single modules' scores (Table 2); also the multiple logistic regression analysis performed between the four different categories of weight status (underweight, normal, overweight, obese) and the three levels of FL (low, medium, high) did not reveal any significant association $(P=0.367)$.

Pearson's $r$ of the overall score with age was 0.44 $(P<0.001)$, so that older children obtained significantly higher scores than younger ones; the same was found in each module, with $r$ ranging from 0.23 to 0.30 (see online supplementary material, Supplemental Table S2). Stratification of the FL overall score into age classes of $\leq 47$ months, 48-59 months and $\geq 60$ months revealed the following significantly different mean (SD) values: 12.2 (4.50), 14.4 (3.66), 17.2 (2.95), $P<0.001$. Weight and height were significantly correlated to the overall score and the score for each module, while BMI was not (Supplemental Table S2).

The multivariate regression showed that independent variables explained $27.3 \%$ of the variability in the FL overall score $\left(R^{2}=0.273\right)$ and the overall regression model was a good fit for the data, as revealed by the $F$-ratio test $\left(F_{(5,437)}=32.89, P<0.001\right)$. This analysis indicated that girls performed better than boys in the overall score, and in Modules 3 and 4 (Table 3). In the multivariate analysis school socio-economic environment was a predictor only for Module 4, with children coming from lower-level

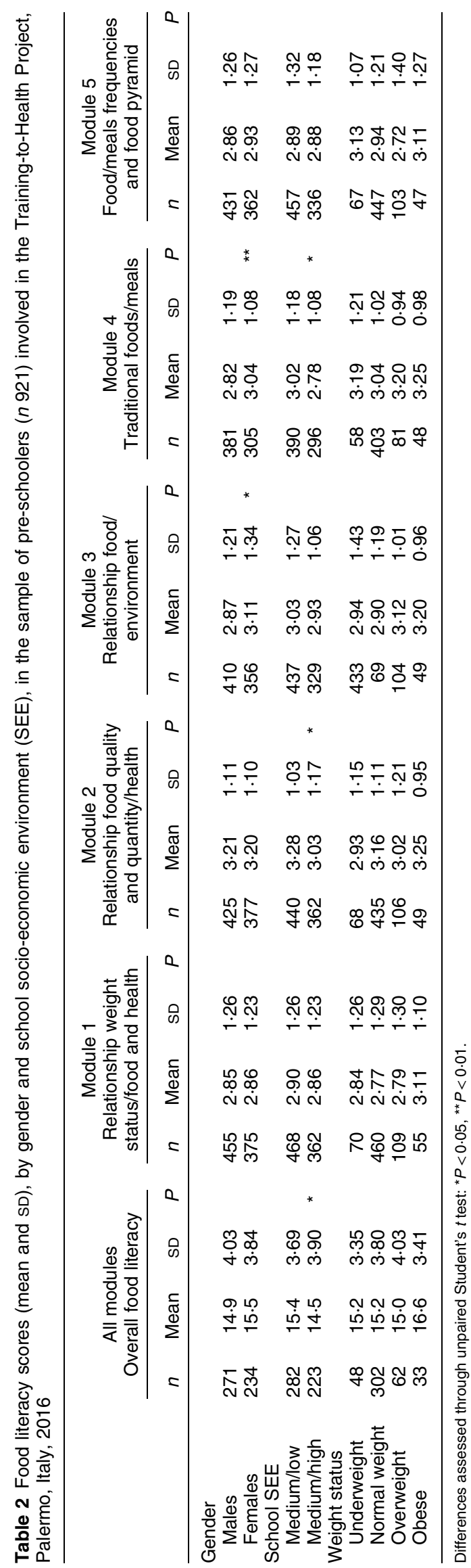


schools performing better than those attending schools of medium/high level $(\beta=-0.33$, $\mathrm{SE}=0.08, P<0 \cdot 001$; Table 3). Multiple linear regression analysis showed that age and height were independent predictors of all modules' scores, while weight was not (Table 3).

The bivariate correlation between QGMD and overall score and each of the module scores was not significant; except that a quite low significant coefficient was found for Module 3 (see online supplementary material, Supplemental Table S3).

In the multivariate regression analysis, the overall FL score was associated with QGMD, even though with a quite low coefficient $(\beta=0.03, P<0.01)$, and in particular it was associated with the component of locomotor skills ( $\beta=0 \cdot 16, P<0 \cdot 001 ;$ Table 4 ). No association was revealed with the control object skills. For the single Modules 1 and 2 , no associations were found in the multivariate analysis.

Almost all the correlations with the emergent literacy skills, instead, were statistically significant; Pearson's $r$ between all emergent literacy skills and overall score ranged from -0.21 to -0.37 (see online supplementary material, Supplemental Table S3). The multivariate analysis showed that lower time on the task of partially hidden objects naming $(\beta=-0.02, P<0.01)$ as well as lower number of errors on the tasks of printed letters identification $(\beta=-0.23, P<0.01)$, objects naming $(\beta=-0.47, P<0.01)$ and partially hidden objects naming $(\beta=-0 \cdot 17, P<0 \cdot 01)$ were determinants of higher FL scores (Table 4).

\section{Discussion}

The present study described FL and its predictors in a sample of pre-school children, highlighting the existing association with gross motor skills and emergent literacy abilities. The authors retain that FL is an important component to be assessed in such early age. Although there is no consensus regarding the definitions, constituents, use or relationship to one another of the terms 'food literacy' and 'nutrition literacy' ${ }^{\text {(36) }}$ (so that these terms are often used interchangeably in public health), in the present study the term 'food literacy' was used instead of 'nutrition literacy' to describe the wide range of skills needed for a healthy and responsible nutrition behaviour, also according to the conceptual clarification of these competing terms recently provided by some authors ${ }^{(37)}$.

A proportion of about $21.0 \%$ of the sample population revealed a high level of FL. The mean scores of the single modules were statistically different. The best mean score (3.2) was found for Module 2 aimed at investigating the relationship between food quality/quantity and health. In this module children demonstrated to have an overall good knowledge of the main food categories, of the different colours of foods, to be able to recognize and name the healthy and non-healthy foods, to be able to weigh foods, to discriminate different quantities (small, medium, big portions), and to understand the relationship between portion and health, and between food colour and health.

A mean score of 3.0 was shown for Module 3, with children understanding quite well the relationship of food/environment, the meaning of 'organic food', recognizing packed organic products and being able to identify food seasonality.

Gross motor development was expressed as a composite score of a set of gross motor skills across locomotor and control object modules. We observed that children had mean locomotor and control object skills of 13.8 (SD 4.29) and 14.0 (SD 3.39), respectively, and a mean QGMD score of $123 \cdot 1$ (SD 19.12) (high motor ability). This is because our correlational descriptive study was carried out within the Training-to-Health Project in which physical education specialists performed an exercise programme that included activities with specific aims of developing fundamental motor and perceptual-sensory skills in children involved in our study. These findings are consistent with data of previous studies that showed a high level of fundamental motor skills in active pre-school children $^{(38,39)}$.

The multivariate regression analysis identified female gender, older age and greater height as independent predictors of a better FL. Girls' score was about 1 point higher than boys'; this is confirmed also in one study on children aged 7-11 years which found that girls achieved $85 \%$ accuracy compared with only $65 \%$ accuracy for boys ${ }^{(40)}$. However, it is not in line with another study that found no gender differences in classifying food as healthy/ non-healthy ${ }^{(6)}$.

As expected, older children performed better than younger ones, with a score increase of about 1 point per each year of age increase. In parallel, an increase of $1 \mathrm{~cm}$ in height increased the overall score by about 1 point. The result relative to height could be due to the effect of age, since height was highly related to age in the present study ( $r=0.63, P<0.001)$, and mean height was significantly higher in boys than girls $(109.6$ v. $107.7 \mathrm{~cm}$, $P<0 \cdot 001)$. Therefore, height could be an indirect predictor of FL.

Age was also an important predictor of each knowledge/skill of the single modules. For each increasing year of age, children had on average an overall score higher by almost 1 point.

It is interesting to note that children attending schools placed in more deprived areas had higher scores in Module 4, which assessed the knowledge and skills relative to traditional foods, including the Mediterranean diet, the typical Sicilian foods and spices (shape, smell and flavour), the ability to manipulate flour and water to obtain a typical food and to assemble a meal by choosing typical foods. It could be hypothesized that children from low socio-economic level know traditional foods better since their families are more connected with 


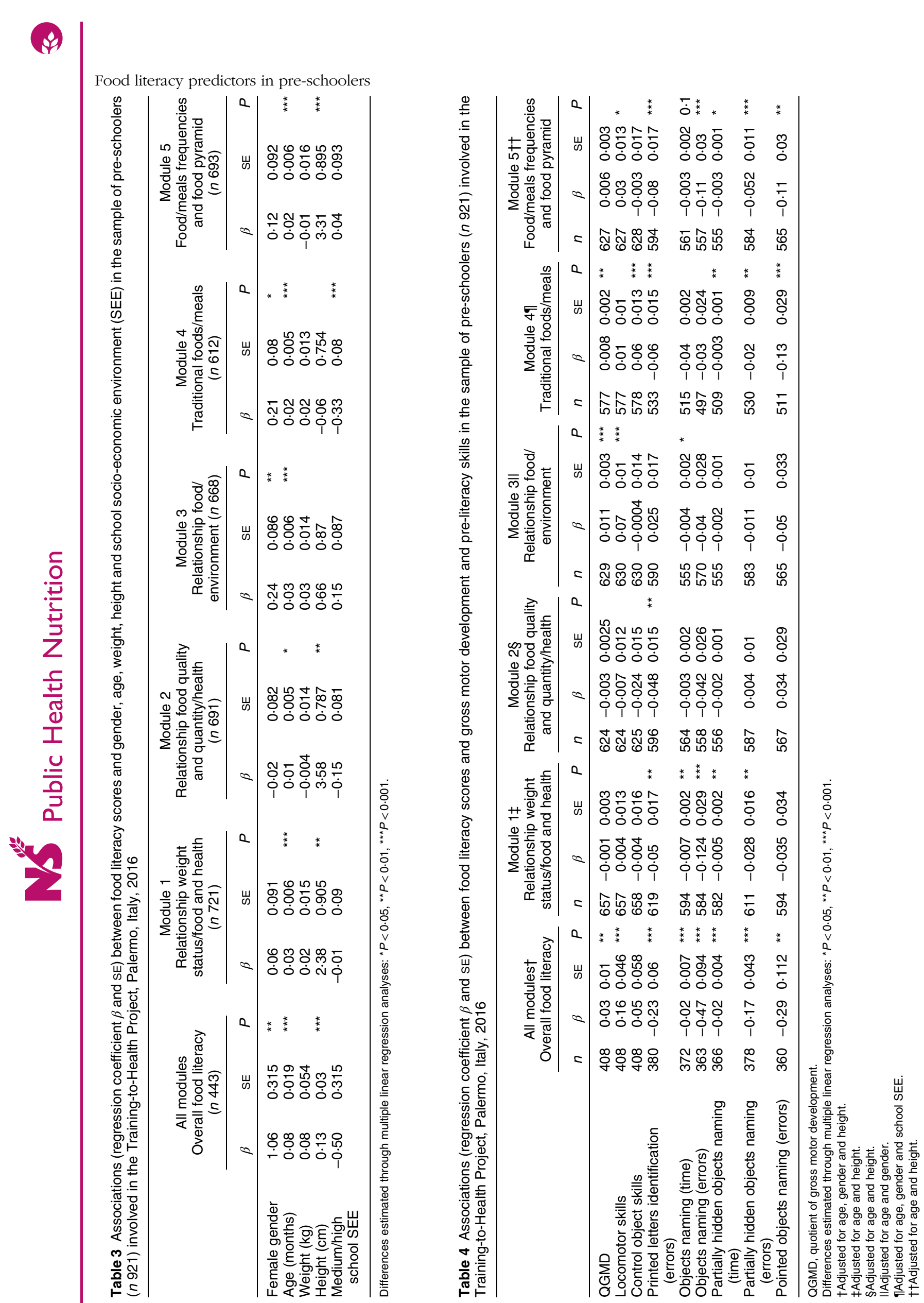


tradition than high-level families, which, instead, are more aware of other products or are used to buying more expensive and less genuine foods such as pre-cooked or frozen foods.

We found that overall FL was associated with the quotient of gross motor development even though with a quite low $\beta$ coefficient and no important association was revealed with the control object skills. Several gaps are present in the literature about the possible association between FL and gross motor development in pre-school children. Moreover, the multivariate regression analysis and quite low $\beta$ coefficient do not allow us to speculate on a possible correlation between FL and fundamental motor skills. Therefore, additional research is needed to study the relationship between FL and motor skills in pre-school children.

All emergent literacy skills were significantly associated with FL scores. Although different studies have investigated emergent literacy skills in children attending kindergarten $^{(12,41,42)}$, insufficient information on the relationship between emergent literacy skills and FL is present in the literature, so a comparison with other studies is difficult. Anyway, the association revealed in the present study is quite intuitive, since very young pre-school children who start developing good pre-reading and pre-writing abilities can be able to better perform activities requiring such skills as the assessment of awareness of healthy/non-healthy foods, the concept of wellness and correct weight status, and all the other aspects investigated through the FL assessment tool described in the present project.

Conversely, sparse research has been produced on the positive association among healthy food consumption and cognitive functioning ${ }^{(43,44)}$. A healthy food style has beneficial effects on student attendance, school grades and general cognitive performances such as speed of processing, attention and concentration, planning and control strategies employed, and working memory. So, a plausible explanation of our result is that, since an early age, children with higher emergent literacy abilities could have received in their families a food education focused on building nutrition knowledge and behaviours. As a consequence, this food education has been translated by their families into a better nutrition and this, as corroborated in the literature, would contribute to a better development of visual analysis, spatial orientation, visual attention and discrimination abilities. However, this is only a conjecture that needs to be addressed in future research.

One limitation could be the response burden that is a critical issue in many of the assessment studies targeted to young children ${ }^{(45)}$. A strength of the present study can be found in the used tool, which was designed to be developmentally appropriate, with the use of pictures, smiles, activities suitable for small children, tangible experiences with food and food products; it targeted different aspects of nutritional knowledge/awareness and of abilities/skills, such as children's ability to recognize and name a wide range of foods, to classify foods according to their healthiness, etc. It is an instrument with good psychometric properties, representing at the moment the only tool available in the literature aimed at assessing FL in pre-schoolers.

\section{Conclusions}

The present study adds important information to the literature regarding FL in pre-schoolers. Previous literature found that FL in the current era is an indispensable need for people because food is connected with every aspect of life including health, culture, personal interest and environment; it is a collection of interrelated knowledge, skills and behaviours required to plan, manage, select, prepare and eat foods to meet needs and determine food intake ${ }^{(8)}$. Children's eating patterns and food preferences are established early in life, therefore the early childhood years are critical for neurocognitive development and for the prevention of diseases related to food, such as obesity, diabetes, CVD and other metabolic disorders. It is recognized that one way of understanding the reasons behind the nutrition-related problems and behaviours among children is by assessing and monitoring their FL level ${ }^{(46)}$ using proper instruments and methodology. Information provided by the present study emphasizes the need of assessing and monitoring FL, together with locomotor, pre-reading and pre-writing skills, which are related to the development of food knowledge and of some food abilities. The results of these actions could assist health and education practitioners to make decisions related to actively promoting healthy eating, education programmes and physical activity in schools since pre-school age, to enhance overall success at school. This is supported by literature stating that raising children in 'enriched environments' induces better learning and memory and greater capacity for plasticity and behavioural adaptation ${ }^{(47)}$; similarly, children raised in environments where both cognitive and motor skills are enhanced can have better chances of developing FL and increasing success at school ${ }^{(48,49)}$.

Future studies aimed at preventing obesity, therefore, should consider academic and cognitive as well as physical outcomes in pre-school settings, and supporting and monitoring FL development.

\section{Acknowledgements}

Acknowledgements: The authors wish to thank Barbara Evola (city councillor), Maria Anna Fiasconaro (municipal manager), Carmen Modica (student who uploaded the data and provided informatic support), schoolteachers and Physical Education graduates for their kind collaboration in this study. Financial support: The study was funded by the Municipality of Palermo (grant number PJ_CT_D12_EDUSAN_0001). The Municipality of Palermo 
had no role in the design, analysis or writing of this article and had no additional role. Conflict of interest: None. Authorship: G.T. drafted the manuscript, analysed and commented on the data. G.B. conducted the training sessions and data acquisition. M.A. participated in the interpretation of data. A. Paoli helped to draft the manuscript. A. Palma participated in the study design. M.B. was responsible for study design, drafting the manuscript and coordination. All authors have read and approved the final version of the manuscript and agree with the order of presentation of the authors. Ethics of human subject participation: This study was conducted according to the guidelines laid down in the Declaration of Helsinki and all procedures involving research study participants were approved by the Ethical Board of the Azienda Ospedaliera Universitaria Policlinico Paolo Giaccone Palermo (Palermo 1, No. 02/2018). Written informed consent was obtained from all children's parents. Trial registration: The project was registered at ClinicalTrials.gov (identifier: NCT03454061, registered: 2 March 2018).

\section{Supplementary material}

To view supplementary material for this article, please visit https://doi.org/10.1017/S1368980019002404

\section{References}

1. Wardle J (1995) Parental influences on children's diets. Proc Nutr Soc 54, 747-758.

2. Khambalia AZ, Dickinson S, Hardy LL et al. (2012) A synthesis of existing systematic reviews and meta-analyses of school-based behavioural interventions for controlling and preventing obesity. Obes Rev 13, 214-233.

3. Larson N, Ward D, Neelon S et al. (2011) Preventing Obesity Among Preschool Children: How Can Child-Care Settings Promote Healthy Eating and Physical Activity? Research Synthesis. Princeton, NJ: Active Living Research, a National Program of the Robert Wood Johnson Foundation. https:// activelivingresearch.org (accessed August 2019).

4. Story M, Kaphingst KM \& French S (2006) The role of schools in obesity prevention. Future Child 16, 109-142.

5. Campbell KJ \& Hesketh KD (2007) Strategies which aim to positively impact on weight, physical activity, diet and sedentary behaviours in children from zero to five years. A systematic review of the literature. Obes Rev 8, 327-338.

6. Zarnowiecki D, Dollman J \& Sinn N (2011) A tool for assessing healthy food knowledge in 5-6-year-old Australian children. Public Health Nutr 14, 1177-1183.

7. Cullen T, Hatch J, Martin W et al. (2015) Food literacy: definition and framework for action. Can J Diet Pract Res $\mathbf{7 6}$, 140-145.

8. Vidgen HA \& Gallegos D (2014) Defining food literacy and its components. Appetite 76, 50-59.

9. Mikkelsen BE, Rasmussen VB \& Young I (2005) The role of school food service in promoting healthy eating at school - a perspective from an ad hoc group on nutrition in schools, Council of Europe. Food Serv Technol 5, 7-15.

10. Fisher D, McDonald N \& Strickland J (2001) Early literacy development: a sound practice. Gen Music Today 14, 15-20.
11. Geertsen SS, Thomas R, Larsen MN et al. (2016) Motor skills and exercise capacity are associated with objective measures of cognitive functions and academic performance in preadolescent children. PLoS One 11, e0161960.

12. Shanahan T \& Lonigan CJ (2010) The national early literacy panel: a summary of the process and the report. Educ Res 39, 279-285.

13. Piek JP, Dawson L, Smith LM et al. (2008) The role of early fine and gross motor development on later motor and cognitive ability. Hum Mov Sci 27, 668-681.

14. Piek JP, Bradbury GS, Elsley SC et al. (2008) Motor coordination and social-emotional behaviour in preschoolaged children. Int J Disabil Dev Educ 55, 143-151.

15. Roebers CM \& Kauer M (2009) Motor and cognitive control in a normative sample of 7-year-olds. Dev Sci 12, $175-181$

16. van der Fels IMJ, te Wierike SCM, Hartman E et al. (2015) The relationship between motor skills and cognitive skills in 4-16 year old typically developing children: a systematic review. J Sci Med Sport 18, 697-703.

17. Diamond A (2000) Close interrelation of motor development and cognitive development and of the cerebellum and prefrontal cortex. Child Dev 71, 44-56.

18. Grosso G, Marventano S, Buscemi S et al. (2013) Factors associated with adherence to the Mediterranean diet among adolescents living in Sicily, southern Italy. Nutrients $\mathbf{5}$, 4908-4923.

19. Fahlman MM, Dake JA, McCaughtry $\mathrm{N}$ et al. (2008) A pilot study to examine the effects of a nutrition intervention on nutrition knowledge, behaviors, and efficacy expectations in middle school children. $J$ Sch Health $\mathbf{7 8}$, 216-222.

20. Hall E, Chai W \& Albrecht JA (2016) Relationships between nutrition-related knowledge, self-efficacy, and behavior for fifth grade students attending Title I and non-Title I schools. Appetite 96, 245-253.

21. Baskale H \& Bahar Z (2011) Outcomes of nutrition knowledge and healthy food choices in 5-to 6-year-old children who received a nutrition intervention based on Piaget's theory. J Spec Pediatr Nurs 16, 263-279.

22. Joseph LS, Gorin AA, Mobley SL et al. (2015) Impact of a short-term nutrition education child care pilot intervention on preschool children's intention to choose healthy snacks and actual snack choices. Child Obes 11, 513-520.

23. Sigman-Grant M, Byington TA, Lindsay AR et al. (2014) Preschoolers can distinguish between healthy and unhealthy foods: the all 4 kids study. I Nutr Educ Behav 46, 121-127.

24. Gibson EL, Wardle J \& Watts CJ (1998) Fruit and vegetable consumption, nutritional knowledge and beliefs in mothers and children. Appetite 31, 205-228.

25. Wiseman N, Harris N \& Downes M (2017) Validation of an iPad activity to measure preschool children's food and physical activity knowledge and preferences. Int J Behav Nutr Phys Act 14, 11.

26. Wiseman N, Harris N \& Lee P (2016) Lifestyle knowledge and preferences in preschool children: evaluation of the get up and grow healthy lifestyle education programme. Health Educ J 75, 1012-1024.

27. Wiseman N \& Harris N (2015) A systematic review of data collection techniques used to measure preschool children's knowledge of food and nutrition. J Nutr Educ Behav $\mathbf{4 7}$, 345-353.e1.

28. Perry EA, Thomas H, Samra HR et al. (2017) Identifying attributes of food literacy: a scoping review. Public Health Nutr 20, 2406-2415.

29. Gibbs H \& Chapman-Novakofski K (2013) Establishing content validity for the nutrition literacy assessment instrument. Prev Chronic Dis 10, 120267. 
30. Rothman RL, Housam R, Weiss H et al. (2006) Patient understanding of food labels. The role of literacy and numeracy. Am I Prev Med 31, 391-398.

31. Weiss BD, Mays MZ, Martz W et al. (2005) Quick assessment of literacy in primary care: the newest vital sign. Ann Fam Med 3, 514-522.

32. Battaglia G, Alesi M, Tabacchi G et al. (2019) The development of motor and pre-literacy skills by a physical education program in preschool children: a non-randomized pilot trial. Front Psychol 9, 2694.

33. Cole TJ, Bellizzi MC, Flegal KM et al. (2000) Establishing a standard definition for child overweight and obesity worldwide: international survey. BMJ 320, 1240-1243.

34. Ulrich DA (1985) Test of Gross Motor Development. Austin, TX: PRO-ED.

35. Cornoldi C, Miato L, Molin A et al. (2009) PRCR-2/2009. Prove di Prerequisito per la Diagnosi delle Difficoltà di Lettura e Scrittura. Firenze: Giunti OS.

36. Velardo S (2015) The nuances of health literacy, nutrition literacy, and food literacy. J Nutr Educ Behav 47, 385-389.

37. Krause C, Sommerhalder K, Beer-Borst S et al. (2018) Just a subtle difference? Findings from a systematic review on definitions of nutrition literacy and food literacy. Health Promot Int 33, 378-389.

38. Derri V, Tsapakidou A, Zachopoulou E et al. (2001) Complexity of rhythmic ability as measured in preschool children. Percept Mot Skills 92, 777-785.

39. Hestbaek L, Andersen ST, Skovgaard T et al. (2017) Influence of motor skills training on children's development evaluated in the Motor skills in PreSchool (MiPS) study-DK: study protocol for a randomized controlled trial, nested in a cohort study. Trials 18, 400.
40. Hart KH, Bishop JA \& Truby H (2002) An investigation into school children's knowledge and awareness of food and nutrition. J Hum Nutr Diet 15, 129-140.

41. Puranik CS \& Lonigan CJ (2011) From scribbles to scrabble: preschool children's developing knowledge of written language. Read Writ 24, 567-589.

42. Pinto G, Bigozzi L, Tarchi C et al. (2016) Predicting reading, spelling, and mathematical skills: a longitudinal study from kindergarten through first grade. Psychol Rep 118 413-440.

43. Hoyland A, Dye L \& Lawton CL (2009) A systematic review of the effect of breakfast on the cognitive performance of children and adolescents. Nutr Res Rev 22, 220-243.

44. Ni Mhurchu C, Turley M, Gorton D et al. (2010) Effects of a free school breakfast programme on school attendance, achievement, psychosocial function, and nutrition: a stepped wedge cluster randomised trial. BMC Public Health 10, 738.

45. Hernández-Garbanzo Y, Brosh J, Serrano EL et al. (2013) Psychosocial measures used to assess the effectiveness of school-based nutrition education programs: review and analysis of self-report instruments for children 8 to 12 years old. J Nutr Educ Behav 45, 392-403.

46. Vaitkeviciute R, Ball LE \& Harris N (2015) The relationship between food literacy and dietary intake in adolescents: a systematic review. Public Health Nutr 18, 649-658.

47. Johnson SB, Riis JL \& Noble KG (2016) State of the art review: poverty and the developing brain. Pediatrics 137, e20153075.

48. Neumann MM (2016) A socioeconomic comparison of emergent literacy and home literacy in Australian preschoolers. Eur Early Child Educ Res J 24, 555-566.

49. Gillon GT (2007) Phonological Awareness: From Research to Practice (Challenges in Language and Literacy). New York: Guilford Publications. Inc. 